\title{
Composición de Ácidos Grasos (MUFA y CLA) en Tejido Muscular de Bovino Relacionado con la Presencia del Polimorfismo g.878TC en el Gen SCD
}

\author{
Fatty Acid Composition (MUFA and CLA) in Bovine Muscle Tissue Related \\ with the Presence of the g.878TC SCD Gene Polymorphism \\ *Inostroza, K.; ${ }^{* *}$ Larama, G. \& ${ }^{* * * *}$ Sepúlveda, N.
}

INOSTROZA, K.; LARAMA, G. \& SEPÚLVEDA, N. Composición de ácidos grasos (MUFA y CLA) en tejido muscular de bovino relacionado con la presencia del polimorfismo g.878TC en el gen SCD. Int. J. Morphol., 30(3):934-936, 2012.

RESUMEN: El tejido muscular de muchos animales domésticos es fuente de proteínas, grasa y minerales para los seres humanos y está compuesto por una serie de estructuras que le otorgan propiedades nutricionales y bioquímicas. En los ultimos años se ha identificado un polimorfismo de único nucleótido (SNP) en el gen SCD (g.878TC), que influye sobre la composición de ácidos grasos en los bovinos. El objetivo de este estudio fue determinar la presencia del SNP g.878TC en músculo Longissimus dorsi de bovino (Bos taurus) por medio de la técnica PCR-RFLP. Fue utilizada una muestra de 100 novillos de raza Aberdeen Angus criados y sacrificados en la Región de La Araucanía (Chile). La frecuencia genotípica del polimorfismo fue de: 0,33 para TT, 0,43 para TC y 0,24 para el genotipo CC. La frecuencia alélica fue de 0,54 para el alelo T y 0,63 para el alelo C. De acuerdo a la composición de ácidos grasos, se determinó que existe una relación positiva entre el genotipo CC y el contenido de ácidos grasos MUFA y CLA presentes en el músculo Longissimus dorsi de novillos Angus. Por lo tanto, el SNP g.878TC podría ser considerado como un marcador genético para la selección de animales Aberdeen Angus, con una composición de ácidos grasos más saludable.

PALABRAS CLAVE: Músculos; Marcadores genéticos; Lípidos; Salud humana.

\section{INTRODUCCIÓN}

La composición de la grasa en la carne bovina tiene gran importancia en la alimentación humana. Se ha asociado la presencia de ácidos grasos saturados tales como el ácido mirístico (C14:0) y ácido palmítico (C16:0) a patologías cardiovasculares en consumidores de carnes rojas (Keys et al., 1974). Por otro lado, el consumo de ácidos grasos polinsaturados y monoinsaturados (PUFA y MUFA) se reporta como esenciales para la dieta humana (Liechtenstein et al., 1998; Kouba \& Mourot, 2011). Últimamente se ha reportado que la carne de vacuno también es un alimento funcional ya que es una fuente importante de ácido linoleico conjugado (CLA), el cual tiene un papel fundamental en la salud humana (Keating et al., 2006).

En los rumiantes la enzima estearoyl CoA desaturasa (SCD) cataliza la biosíntesis de ácidos grasos monoinsaturados, originando la síntesis endógena del princi- pal isómero de ácido linoleico conjugado (CLA) cis-9 trans11 CLA (Griinari et al., 2000). Taniguchi et al. (2004), identificaron 8 SNPs en el gen SCD, dentro de los cuales se menciona principalmente el SNP g.878TC, el cual causa una sustitución de aminoácidos de valina a alanina en la proteína.

El objetivo de este estudio fue determinar la relación del polimorfismo g.878TC con la composición de ácidos grasos MUFA y CLA en músculo Longissimus dorsi de vacunos de la raza Angus.

\section{MATERIAL Y MÉTODO}

Detección del SNP g.878TC. El estudio fue realizado con 100 novillos de la raza Aberdeen Angus criados en la Nove-

* Programa de Doctorado en Ciencias mención Biología Celular y Molecular Aplicada. Facultad de Ciencias Agropecuarias y Forestales. Universidad de La Frontera, Temuco, Chile.

** Licenciado en Biotecnología, Universidad de La Frontera, Temuco, Chile.

**** Departamento de Producción Agropecuaria. Facultad de Ciencias Agropecuarias y Forestales. Universidad de La Frontera, Temuco, Chile.

Este estudio fue parcialmente financiado con los aportes del Programa FIC-FIA No 2007-009 y Fondecyt 1120474. 
na Región de La Araucanía (Chile) y sacrificados en la Planta Faenadora Temuco en mayo de 2011 con una edad promedio de 12 meses y con un peso de carcaza de $263 \pm 18 \mathrm{~kg}$ en promedio. Dos días después del sacrificio se recolectó una muestra de $100 \mathrm{~g}$ del músculo Longissimus dorsi izquierdo, obtenida de un corte sagital realizado en la porción central (a nivel de la vertebra T10) y se almacenaron posteriormente a $-80{ }^{\circ} \mathrm{C}$ (Cañeque \& Sañudo, 2000). Posteriormente se extrajo ADN genómico de cada muestra con el kit comercial ISOLATE genomic DNA mini Kit (Bioline, EUA).

La presencia del SNP g.878TC se determinó mediante la técnica PCR-RFLP. Se diseñó un set de partidores basados en la secuencia disponible en el GenBank $\left(\mathrm{N}^{\circ}\right.$ de acceso: AF285607): 5'-ACCTGGCTGGTGAATAGTGCT-3' y 5'-TCTGGCACGTAACCTAATACCCT-3', con los cuales se amplificó una región de 170pb del gen SCD. La reacción de PCR se realizó con la enzima Paq5000 DNA Polimerase (Agilent Technologies, EUA) y sometida a un programa de temperatura que incluyó: denaturación inicial $\left(94^{\circ} \mathrm{C}\right.$ por $\left.3 \mathrm{~min}\right)$ y 30 ciclos de amplificación con denaturación $\left(95^{\circ} \mathrm{C}\right.$ por $\left.20 \mathrm{seg}\right)$, hibridación $\left(61^{\circ} \mathrm{C}\right.$ por 20 seg), extensión $\left(72^{\circ} \mathrm{C}\right.$ por $\left.30 \mathrm{seg}\right)$ y extensión final $\left(72^{\circ} \mathrm{C}\right.$ por $5 \mathrm{~min}$ ). Los productos PCR fueron digeridos con la enzima de restricción SatI (Fermentas, EUA), utilizando 2,5 $\mathrm{U}$ de enzima y $10 \mu \mathrm{l}$ de producto de PCR. El mix de digestión fue incubado a $37^{\circ} \mathrm{C}$ toda la noche.

Análisis del contenido de ácidos grasos. Los ácidos grasos de las muestras de músculo L. dorsi, fueron extraídos por el método descrito por Folch et al. (1951). Los ésteres metílicos de ácidos grasos fueron analizados en un cromatógrafo de gases modelo Clarus 500 (Perkin Elmer, EUA), acoplado a un detector de ionización de llama (FID) y equipado con una columna SPTM Fused Silica Capillary Column 2380 (60 m x $0,25 \mathrm{~mm}$ x 0,2 $\mu \mathrm{m}$ film thickness, Supelco, EUA). Para la detección de los ácidos grasos se diseñó un programa de temperaturas de 48 minutos que incluyó: $150^{\circ} \mathrm{C}$ por $1 \mathrm{~min}$, luego un aumento de temperatura a razón de $1^{\circ} \mathrm{C}$ por min hasta llegar a $\operatorname{los} 168^{\circ} \mathrm{C}$ y mantenidos por $11 \mathrm{~min}$, seguido por un segundo incremento de temperatura de $6^{\circ} \mathrm{C}$ por min, para alcanzar finalmente los $230^{\circ} \mathrm{C}$ mantenidos por $8 \mathrm{~min}$. Se utilizó la mezcla estándar de 37 componentes de ácidos grasos F.A.M.E Mix (C4-C24, Supelco, EUA) para la identificación de los ácidos grasos. Además, del estándar Octadecadienoic, Acid, Conjugated Methyl Ester (CLA Sigma, EUA) para la identificación de los isómeros de ácido linoleico conjugado. Todos los ácidos grasos fueron cuantificados mediante curvas de calibración, utilizando el ácido graso Methyl Nonadecanoate (Sigma, EUA) como estándar interno.

Análisis estadístico. Las medias marginales estimadas ( \pm EE) fueron determinadas utilizando un modelo lineal general (SPSS versión 17.0 for Windows), que incluye el efecto fijo del genotipo, edad y peso. Los valores promedio fueron sometidos a test de comparaciones múltiples (Tukey) y determinación de diferencias a $\mathrm{P}<0,05$.

\section{RESULTADOS Y DISCUSIÓN}

Las frecuencias genotípicas y alélicas se describen en la Tabla I. La frecuencia del alelo C del SNP g.878TC se encuentra en el rango mencionado por Taniguchi et al. y Milannesi et al. (2008) en sus estudios realizados con ganado de carne.

Castillo et al. (2010) mencionan que existe un efecto de la variabilidad genética sobre la cantidad total de ácidos grasos en animales manejados con una misma dieta, sin embargo, no puede explicar algún factor genético sobre la dispersión de valores de CLA. El SNP g.878TC está relacionado positivamente con el contenido de ácidos grasos monoinsaturados, y afecta significativamente la cantidad de ácidos grasos miristoleico (C14:1), oleico (C18:1) y CLA (Taniguchi et al.; Mele et al., 2007). En este estudio, los animales con el genotipo CC (Tabla II) poseen un mayor contenido total de ácidos grasos monoinsaturados (MUFA) que los animales con genotipo TT $(\mathrm{P}<0,05)$. Asimismo, altos valores de CLA están vinculados a la presencia del genotipo $\mathrm{CC}$, al igual que los ácidos grasos miristoleico (C14:1), palmitoleico (C16:1), cis-10-heptadecanoico (C17:1), elaídico (C18:1n9t) y oleico (C18:1n9c).

Se puede concluir que la composición de ácidos grasos en músculo Longissimus dorsi en novillos Angus, se encuentra influenciada por polimorfismos presentes en genes relacionados con la síntesis de ácidos grasos. También queda establecido que el SNP g.878TC puede ser un posible marcador genético para la selección de animales con mayores contenidos de MUFA y CLA, y por lo tanto, una composición de ácidos grasos más saludable.

Tabla I. Distribución de los genotipos del polimorfismo 878TC del gen SCD presentes en novillos de raza Angus ( $\mathrm{n}=100)$.

\begin{tabular}{llcc}
\hline Variable & \multicolumn{3}{c}{ Genotipos SNP g.878TC } \\
& TT & TC & CC \\
\hline Frecuencia genotípica & 0,33 & 0,43 & 0,24 \\
\hline Frecuencia alélica & $\mathrm{T}=0,54$ & $\mathrm{C}=0,46$ &
\end{tabular}


Tabla II. Relación del SNP g.878TC con el contenido de ácidos grasos del músculo Longissimus dorsi de novillos Angus. (mg ácido graso/g de músculo).

\begin{tabular}{lrrr}
\hline Ácido Graso & \multicolumn{1}{c}{ TT } & \multicolumn{1}{c}{ TC } & \multicolumn{1}{c}{ CC } \\
\hline C14:1 $14,25 \pm 0,03 \mathrm{~b}$ & $0,33 \pm 0,03 \mathrm{~b}$ & $0,58 \pm 0,04^{\mathrm{a}}$ \\
C16:1 & $1,15 \pm 0,22 \mathrm{~b}$ & $1,61 \pm 0,19 \mathrm{~b}$ & $3,36 \pm 0,25^{\mathrm{a}}$ \\
C17:1 & $0,12 \pm 0,02 \mathrm{~b}$ & $0,19 \pm 0,02 \mathrm{~b}$ & $0,42 \pm 0,03^{\mathrm{a}}$ \\
C18:1n9t & $0,49 \pm 0,20 \mathrm{~b}$ & $1,03 \pm 0,17 \mathrm{~b}$ & $1,81 \pm 0,23^{\mathrm{a}}$ \\
C18:1n9c & $8,42 \pm 1.23 \mathrm{c}$ & $12,41 \pm 1,06 \mathrm{~b}$ & $23,36 \pm 1,41^{\mathrm{a}}$ \\
CLA 1 & $0,16 \pm 0.06 \mathrm{~b}$ & $0,33 \pm 0,05 \mathrm{~b}$ & $0,83 \pm 0,07^{\mathrm{a}}$ \\
MUFA $^{2}$ & $10,43 \pm 1,47 \mathrm{c}$ & $15,57 \pm 1,27 \mathrm{~b}$ & $29,53 \pm 1,69^{\mathrm{a}}$ \\
MUFA: SFA $^{3}$ & $0,80 \pm 0,02 \mathrm{~b}$ & $0,88 \pm 0,02 \mathrm{a}$ & $0,92 \pm 0,02^{\mathrm{a}}$ \\
\hline
\end{tabular}

1CLA: cis-9, trans-11/trans-9 cis-11; 2MUFA: Monounsaturated fatty acids; 3SFA: Saturated fatty acids.

AGRADECIMIENTOS. Los autores agradecen al Frigorífico Temuco S.A. por la disposición de las muestras analizadas en estudio. Este trabajo es parte de la tesis de postgrado de la Ing. Agrónomo Karla Inostroza apoyada por CONICYT a través de una beca de apoyo para Tesis Doctoral.

INOSTROZA, K.; LARAMA, G. \& SEPÚLVEDA, N. Fatty acid composition (MUFA and CLA) in bovine muscle tissue related with the presence of the g.878TC SCD gene polymorphism. Int. J. Morphol., 30(3):934-936, 2012.

SUMMARY: The muscle tissue of many domestic animals is a source of protein, fat and minerals to humans and consists of a series of structures that give nutritional and biochemical properties. In recent years it has identified a single nucleotide polymorphism (SNP) in the SCD gene (g.878TC), which influences the fatty acid composition in cattle. The aim of this study was to determine the presence of SNP g.878TC in Longissimus dorsi bovine (Bos taurus) using the PCR-RFLP. It was used a sample of 100 Aberdeen Angus steers raised and slaughtered in the region of Araucania (Chile). Polymorphism genotype frequency were: 0.33 for TT, 043 for $\mathrm{CT}$ and 0.24 for the CC genotype. The allelic frequency was 0.54 for the $\mathrm{T}$ allele and 0.63 for allele $\mathrm{C}$. According to the fatty acid composition was determined that a positive relationship between genotype CC and the content of MUFA and CLA fatty acids present in the Longissimus dorsi muscle of Angus steers. Therefore, the SNP g.878TC could be considered as a genetic marker for selection of animals Aberdeen Angus, with a fatty acid composition more healthy.

KEY WORDS: Muscle; Genetic markers; Lipids; Human health.

\section{REFERENCIAS BIBLIOGRÁFICAS}

Cañeque, V. \& Sañudo, C. Metodología para el estudio de la calidad de la canal y de la carne en ruminates. Madrid, España, Instituto de Investigación y Tecnología Agraria y Alimentaria, 2000. p.254.

Castillo, J.; Pabón, M.; Olivera, M. \& Carulla, J. Role of stearoyl CoA desaturase on conjugated linoleic acid concentration in bovine milk: review. Rev. Colom. Cienc. Pecu., 23:493-500, 2010.

Folch, J.; Ascoli, I.; Lees, M.; Meath, J. \& Lebaron, F. Preparation of lipids extracts from brain tissue. J. Biol. Chem., 191(2):833-41, 1951.
Griinari, J. M.; Corl, B. A.; Lacy, S. H.; Chouinard, P. Y.; Nurmela, K. V. \& Bauman, D. E. Conjugated linoleic acid is synthesized endogenously in lactating dairy cows by Delta(9)-desaturase. $J$. Nutr., 130(9):2285-91, 2000.

Keating, A. F.; Kennelly, J. J. \& Zhao, F. Q. Characterization and regulation of bovine stearoyl CoA desaturase gene promoter. Biochem. Biophys. Res. Commun., 344(1):233-40, 2006.

Keys, A.; Grande, F. \& Anderson, J. Bias and misrepresentation revised: perspective on saturated fat. Am. J. Clin. Nutr., 27:188212, 1974.

Kuoba, M. \& Mourot, J. A review of nutritional effects on fat composition of animal products with special emphasis on $\mathrm{n}-3$ polyunsaturated fatty acid. Biochimie, 93(1):13-7, 2011.

Lichtenstein, A. H.; Kennedy, E.; Barrier, P.; Danford, D.; Ernst, N. D.; Grundy, S. M.; Leveille, G. A.; Van Horn, L.; Williams, C. L. \& Booth, S. L. Dietary fat consumption and health. Nutr. Rev., 56(5 Pt 2):S3-19; discussion S19-28, 1998.

Mele, M.; Conte, G.; Castiglioni, B.; Cheessa, S.; Macciottan, N.; Serra, A.; Buccioni, A.; Pagnacco, G. \& Secchiari, P. Stearoyl-coenzyme A desaturase gene polymorphism and milk fatty acid composition in Italian Holsteins. J. Dairy Sci., 90(9):4458-65, 2007.

Milannesi, E.; Nicoloso, L. \& Crepaldi, P. Stearoyl CoA desaturase (SCD) gene polymorphism in Italian castle breeds. J. Anim. Breed Genet., 125(1):63-7, 2008.

Taniguchi, M.; Utsugi, T.; Oyama, K.; Mannen, H.; Kobayachi, M.; Tanabe, Y.; Ogino, A. \& Tsuji, S. Genotype of stearoyl CoA desaturase is associated with fatty acid composition in Japanese black cattle. Mamm. Genome, 14(2):142-8, 2004.

Dirección para correspondencia:

Inostroza, K

Programa de Doctorado en Ciencias mención Biología

Celular y Molecular Aplicada

Facultad de Ciencias Agropecuarias y Forestales

Universidad de La Frontera, Temuco

CHILE

Email: karla.inostroza@gmail.com Recibido: 19-06-2012

Aceptado: 08-08-2012 